\title{
2 Data on losses for risk evaluation
}

Historical loss data is of key importance for risk management and modeling of losses. This statistical information must be carefully analyzed and understood in order to extract the best possible knowledge. Therefore, it is important to collect details on the data sources, such as framework, time of collection, definitions, exceptions and so on.

Insurance companies have always been collecting data for risk management purposes, but in the past information was rather aggregate whereas nowadays, there is much more detailed knowledge on individual policy holders, events, claims handling and loss compensations. Nevertheless, data quality still remains a challenge for many insurers and the lack of robustness of statistical information may sometimes pose huge problems to the undertaking of ambitious risk management initiatives.

This chapter presents examples of loss data, which are used throughout some parts of this book. They are intended to be helpful to understand concepts. They do not intend to represent any particular insurance company.

Thereafter, we continue by discussing several characteristics of the loss severity information that has been specially created for this book and their aggregation by means of their sum. This analysis involves statistical description in this chapter and, in the following chapters, we address the implementation of risk measurement and capital allocation. Having a good understanding of amounts of losses is important not only for modeling purposes, but also to identify effective management and mitigating actions.

\subsection{An example on three dimensional data}

Historical loss information is the main source of knowledge for the specific experience and history of the organization. Sources of information on loss 
events that occurred in comparable companies can sometimes be helpful to understand their position with respect to exposure and their corresponding risk. It is sometimes also useful to compare lines of business or business units within a single company.

Throughout most chapters of this book the same data set will be examined. The data consist of three sources of loss and the sum of them. We make use of an artificial sample to provide with a guided path to implementation. Because of the loss amounts have been artificially generated they do not have monetary dimension other than 'monetary units'. Nevertheless, in several parts of the book we consider that these loss amounts are the cost of claims in thousands of euros for three types of damage, let us say property damage, bodily injuries and medical expenses. Table 2.1 and Table 2.2 show some statistical characteristics of the sample, and Figure 2.1 presents a visual view of the three variables and their sum.

There are some interesting features about the example data that are presented in this chapter. All the variables are asymmetric with a few large values and many small values. The maximum values for both $X_{1}$ and $X_{2}$ are much higher than the average. The minimum values are always strictly positive. We also note that the minimum of the sum is not the sum of the minimum and this is due to the fact that the data are paired, and the minimum of each marginal does not necessarily correspond to the minimum observed value of the other marginal.

When comparing the data on Figure 2.1, we note that the size of the losses and the shape of the histograms are similar between sources $X_{1}$ and $X_{2}$ but not between them and source $X_{3}$. Additionally, we cannot see the dependence patterns between the sources unless we look into the bivariate plots. The joint behavior is presented in Figure 2.2.

Table 2.1 Statistical summary of the example loss data

\begin{tabular}{lcccc}
\hline Data & Mean & Median & Std. Deviation & Maximum \\
\hline$X_{1}$ & 0.707 & 0.033 & 3.778 & 49.812 \\
$X_{2}$ & 0.450 & 0.033 & 3.369 & 52.129 \\
$X_{3}$ & 0.268 & 0.105 & 0.361 & 1.775 \\
$X_{1}+X_{2}+X_{3}$ & 1.426 & 0.221 & 5.6797 & 103.716 \\
\hline
\end{tabular}

There are 350 losses for each variable 
Table 2.2 Statistical summary of the example loss data (part II)

\begin{tabular}{lcccc}
\hline Data & Minimum & Variance & Skewness & Kurtosis \\
\hline$X_{1}$ & 0.001 & 14.273 & 8.875 & 94.353 \\
$X_{2}$ & 0.001 & 11.350 & 12.401 & 171.107 \\
$X_{3}$ & 0.003 & 0.130 & 2.119 & 4.296 \\
$X_{1}+X_{2}+X_{3}$ & 0.026 & 46.199 & 11.354 & 153.368 \\
\hline
\end{tabular}

There are 350 losses for each variable

Figure 2.1 Histograms of loss data originating from sources $X_{1}, X_{2}, X_{3}$ and their sum

$\mathrm{X} 1$

$\mathrm{x} 2$
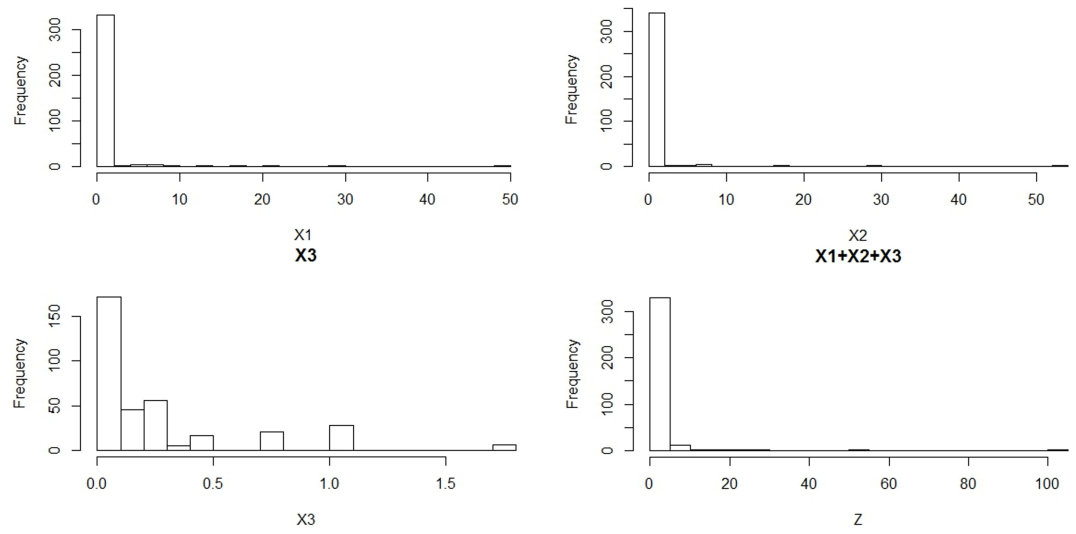

\subsection{Basic graphical analysis of the loss severity distributions}

We can measure risk because different outcomes occur with different probabilities. Loss Distribution Analysis (LDA) is a method used to calculate and evaluate the risk that is induced by losses that occur randomly. Historical data can be helpful to predict future behavior, and so it is important to analyze its density shape.

Modeling the severity of losses usually involves analysis of parametric distributions such as the Normal distribution and all other distributions that 
Figure 2.2 Dependence from sources $X_{1}, X_{2}, X_{3}$ and their sum

\section{Simple Scatterplot Matrix, Z=X1+X2+X3}

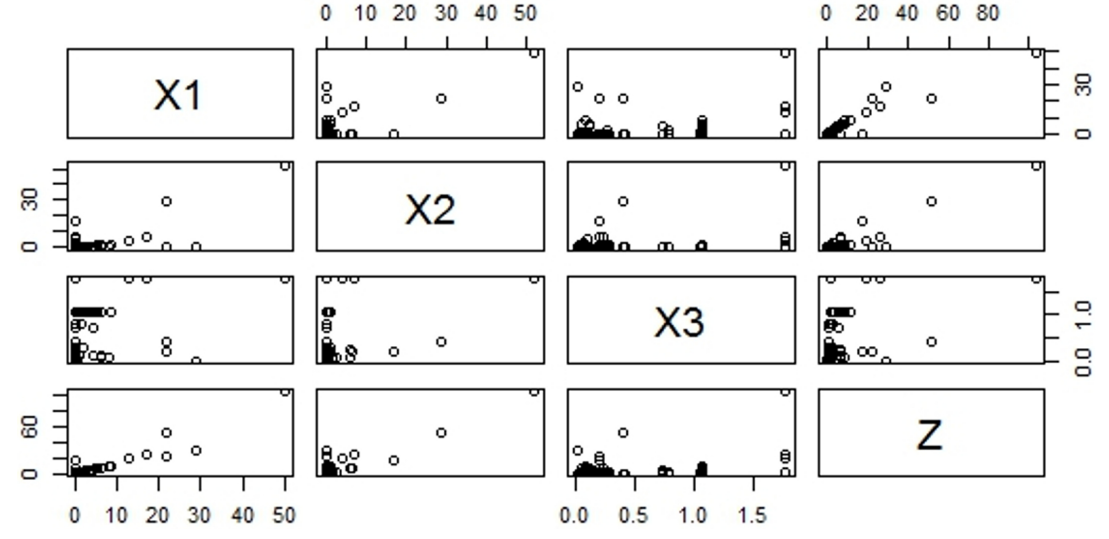

have been mentioned in the previous chapter. However, it is very difficult to find a parametric distribution that fits all sizes of loss: (i) low severities occur frequently, (ii) high severities occur much less often, and (iii) catastrophic losses only occur very rarely. They are usually referred as rare losses or rare events.

This section presents the analysis of one parametric distribution that can be used to fit the behavior observed in the sample data. Some other basic parametric distributions for severity not considered here were presented in the previous chapter.

We have estimated the Normal densities using maximum likelihood in all variables, $X_{1}, X_{2}, X_{3}$ and the sum of the three. Some results are shown in Figures 2.3 to 2.5 . In there, we present the parametric Normal pdf estimate and the original data, the former appearing as dashed lines. On the left, the plots show the lower values of the observed sample and on the right the higher observed values are presented. Low and high values are split in order to see the parts of the densities where data are scarce. Only a simple parametric density estimate is presented in this chapter, but practitioners often use more sophisticated distributions. The empirical distribution function obtained for the sample data is also a possibility, and in this case it is not necessary to fit any distribution in particular.

Figures 2.3 to 2.5 show the maximum likelihood estimation of the Normal densities for the data. The right hand side tail is presented. The density may still be too low for large losses. 
Figure 2.3 The estimated density for the $X_{1}$ data using the Normal distribution
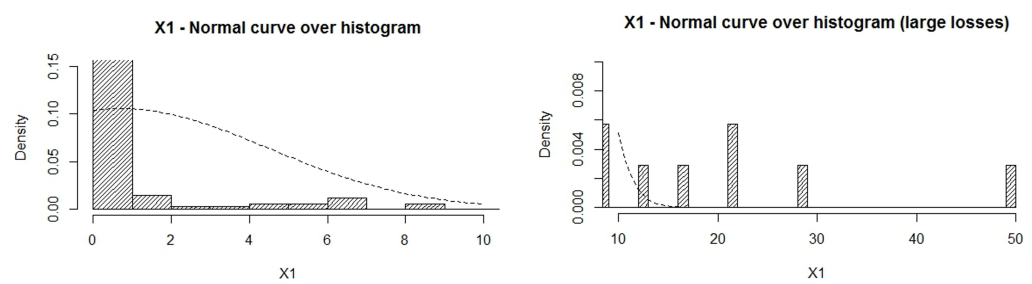

Figure 2.4 The estimated density for the $X_{2}$ data using the Normal distribution
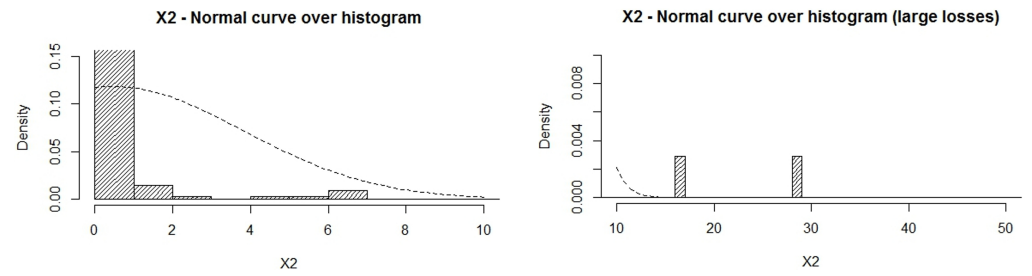

Figure 2.5 The estimated density for the $X_{3}$ data using the Normal distribution
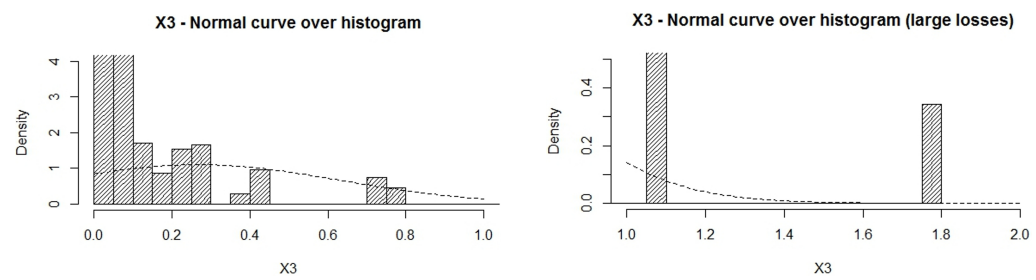

\subsection{Quantile estimation}

Quantile estimation for the parametric distribution presented in the previous section, other distributions or the empirical distribution functions can be performed using the inverse of the distribution function evaluated at the maximum likelihood parameter estimates. This is one of the most basic risk assessment tools that will be used throughout this book and, if one think about Figure 1.2, this is the sort of tools necessary to move from theory to practice.

\subsection{Examples}

Illustrative examples of quantitative analysis are developed in the programming language $\mathrm{R}$. The dataset used in this Chapter (exampleERM) is avail- 
able at http://www.ub.edu/rfa/ERM.

1. Download and save the data in an appropriate directory. Prepare the data:

example $<$ - read.table("exampleERM.txt",header=TRUE)

riskloss $<-$ as.matrix $($ example $)$

2. Descriptive analysis in R. The number of observations and a set of descriptives can be computed as follows:

$\mathrm{n}<-\operatorname{nrow}($ riskloss $)$

summary(riskloss)

E.di $<-$ colMeans(val.di)

Cov.di $<-\operatorname{var}($ val.di $)$

library(moments)

skewness(riskloss[,"Xı"])

kurtosis(riskloss[,"X1"])

3. Graphical analysis in R. Compute the histogram of $\mathrm{X}_{1}$ and scatterplots as follows:

hist(riskloss[,"Xı"], 20)

lines(density(riskloss[,"Xı"]),lwd=3)

plot(riskloss[ ,"Xı"], type="l", ylim=c(o,10))

lines (riskloss[,"X2"], lty=2)

lines(riskloss[ ,"X3"], lty=3)

4. Compute the $\operatorname{VaR}_{90 \%}(X 1)$ and $\mathrm{CTE}_{90 \%}(X 1)$

risk $1<-$ riskloss[,1]

Erisk1 $<-\operatorname{mean}($ risk1)

Vrisk1 $<-\operatorname{var}($ risk 1$)$

VaRgo $<-$ quantile(risk1,o.9o)

CTE9o $<-$ mean(risk1[riskı $>$ VaRgo]) 